\title{
Nanoparticle Quantum Dots in Liquid phase (e.g. CdSe) - Synthesis, Thermodynamical study and Applications
}

\author{
Vikas Sharma ${ }^{1}$ \\ ${ }^{I}$ Centre for Converging Technologies, University of Rajasthan, Jaipur-302004, INDIA
}

\begin{abstract}
Quantum Dots now days have revolutionized the world. These are the nanoparticles having a size less than or around $10 \mathrm{~nm}$. In this paper, an effort has been made to understand the general synthesis of the quantum dots and the thermo dynamical study of the quantum dot particles. The whole process of nucleation and growth is also explained in detail. Various applications of these quantum dots in the different fields of science and technology also been described.
\end{abstract}

Keywords: Bottom-Up Approach, Crystallization, Growth, Nucleation, Supersaturation, Top-Down Approach, Quantum Dot

\section{Introduction}

In the present era is a considerable increase is seen in the interest of the people for the production of nanoparticles. Different methods are employed for nanoparticles' production like chemical methods, physical methods, co-precipitation methods etc. Here in this study we will go through the liquid phase synthesis of nanoparticles, reasons for their preparation and their properties to some extent. This liquid phase synthesis process leads to formation of different structures like quantum dots, quantum wells quantum rings etc. This is the reason why we are interested in this field of study. These prepared structures have wide applications in the field of Biotechnology and others.

\section{EXPLANATION AND DISCUSSION}

2.1 Why nanomaterial quantum dots are so interesting to consider?

They are small in size. Properties depend on size of the particles and can be engineered on the basis of demand. Surface to volume ratio is high and hence provide ample surface area to act upon. Quantum mechanical changes can be done by altering optical and electronic properties. Changes in optical properties may lead to standing waves formation in nanowires, effective waveguiding and strong light-matter interactions. Changes in physical properties like melting point, solubility and hardness provides a large space for improvement.

\subsection{Quantum Dots- Properties}

In Bulk electrons are fully delocalized but in Quantum dots electrons are confined in all the three dimensions. Quantum size effects are experienced:- Band gap increases for smaller particles and discrete energy levels emerge at band edges.(Fig. 1)It is considered as an artificial atom in which an electron remains confined in its level. Density of states represents single line distribution

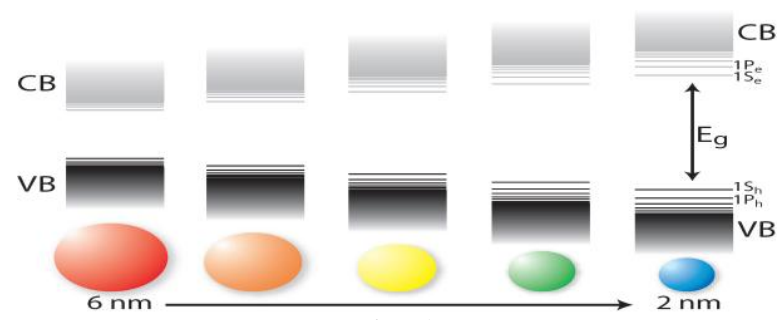

Fig. 1

\subsection{Basic applications of quantum dot nanoparticles}

Solar Cells, Light Emitting Diode, Displays, Low Threshold Lasers, Ultra sensitive Detectors, Ultra photonic Switches, Sensors, Thin Film Field Effect Transistor, Biomedical applications-probes and labels, Photodynamic therapy etc. 
Nanoparticle Quantum Dots in Liquid phase (e.g. CdSe) - Synthesis, Thermodynamical study and

2.4 Approaches to make inorganic nanoparticles (quantum dots)

2.4.1 Top-down approach-

Take the large particles prepared and make them smaller physically. The basic and the most approach to do this is BALL-MILLING Approach.

\subsubsection{Bottom -Up approach-}

- Self assembly of "molecules" into larger structures

- Dominated by crystallization Process.

- Benefits: cheap, larger volumes, very small structures.

- Down side- less control over design

\subsection{Synthesis of CdSe Quantum Dots}

2.5.1 Chemicals Required

Dimethyl Cadmium, Trioctylphosphine selenium

\subsubsection{Method:-}

Take equal amounts of Di-Methyl Cadmium (Reactant A) and Trioctylphosphine selenium (Reactant C) and mix them in a new beaker. Now take another beaker in which highly heated Trioctylphosphine oxide solvent. Now pour the mixed solutions of Reactant A and B into the hot solution of Trioctylphosphine oxide solvent. The injection is done at a temperature of 300 degree Celsius. The capping agent used in this process is TOPO [1]. Reaction taken place is-

$\mathrm{xCdMe}_{2}+\mathrm{xTOP}-\mathrm{Se} \longrightarrow[\mathrm{CdSe}]_{2}+\ldots \ldots$.

When the injection is done completely then the reaction takes place at $300{ }^{\circ} \mathrm{C}$. Now, the temperature comes down and come to $240 \mathrm{C}[2,3]$. Then the further growth of the particles thus formed by the reaction takes place at this temperature from about $30 \mathrm{~min}$ to 2 hours. At this temperature and due to the presence of TOPO as a capping agent monodispersed particles can be obtained [45]. The reaction continues until the temperature reaches to RT value i.e. 82 times of $\mathrm{T}$ and then the reaction stops.

\subsection{Reason for the process of crystallization to occur}

- $\mathrm{G}_{\text {crystal }}<\mathrm{G}_{\text {solution }} \quad$ (where $\mathrm{G}=$ Gibbs energy)

- $\mathrm{G}=\sum \mu_{\mathrm{i}} \mathrm{N}_{\mathrm{i}} \quad\left(\right.$ where $\mu={\text { concentration })^{\mathrm{i}}}^{\mathrm{i}}$

- At equilibrium- $\mu_{\text {crystal }}=\mu_{\text {equilibrium }}$

- $\Delta \mathrm{G}=\Delta \mathrm{H}-\mathrm{T} \Delta \mathrm{S}$ where $\Delta \mathrm{H}=$ enthalpy of crystallization and $\Delta \mathrm{S}=$ entropy of crystallization

- $\Delta \mathrm{H}<0$ : there are more (binding) interactions in the crystal phase

- than in the solution phase

\subsection{Study of Crystallization through $\mathrm{NaCl}$ (generalized)}
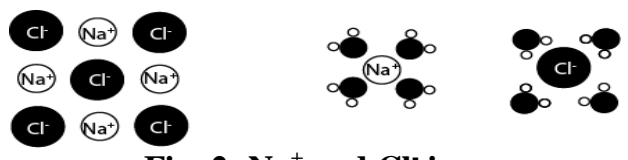

Fig. 2: $\mathrm{Na}^{+}$and $\mathrm{Cl}^{-}$ions

$T_{\text {crystallize }}=\mathrm{K}_{\mathrm{c}}\left[\mathrm{Na}^{+}\right]\left[\mathrm{Cl}^{-}\right] \operatorname{Exp}{ }^{\propto}\left[-\Delta \mu_{\mathrm{c}} / \mathrm{kT}\right]\left(\right.$ where $\mathrm{K}_{\mathrm{c}}=$ coefficient of crystallization $)$

$T_{\text {dissolve }}=\mathrm{K}_{\mathrm{d}}[\mathrm{NaCl}]_{\text {surface }} \approx \operatorname{Exp}\left[-\Delta \mu_{\mathrm{d}} / \mathrm{kT}\right]$

( where $K_{d}=$ dissolving coefficient )

Above two equations can be used to determine the enthalpy crystallization.

\subsection{Supersaturation}

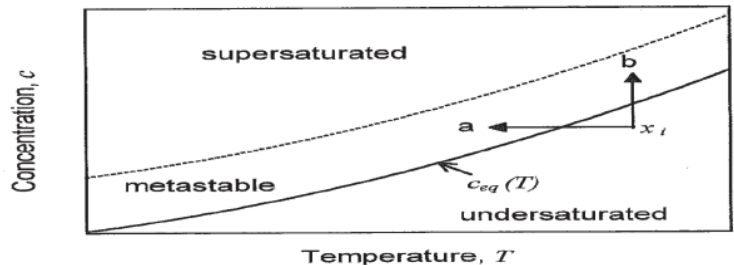

Fig. 3: supersaturation curve 
Supersaturation is the condition at which the concentration of solute reaches to such a level at which no further addition of the solute in the solution takes place and the solute starts aggregating or it starts depositing at the bottom of the container $[7,8]$. :

$\mu_{\mathrm{ss}}>\mu_{\mathrm{eq}}$

Above equation shows that the concentration at supersaturation of the solute is greater than the equilibrium concentration.

$$
\sigma=\left(\mu_{\mathrm{ss}}-\mu_{\mathrm{eq}}\right) / \mathrm{kT}
$$

( where $\sigma=$ supersaturation concentration, $\mathrm{k}=$ Boltzmann's constant, $\mathrm{T}=$ temperature)

\subsection{Free Energy of Crystallization}

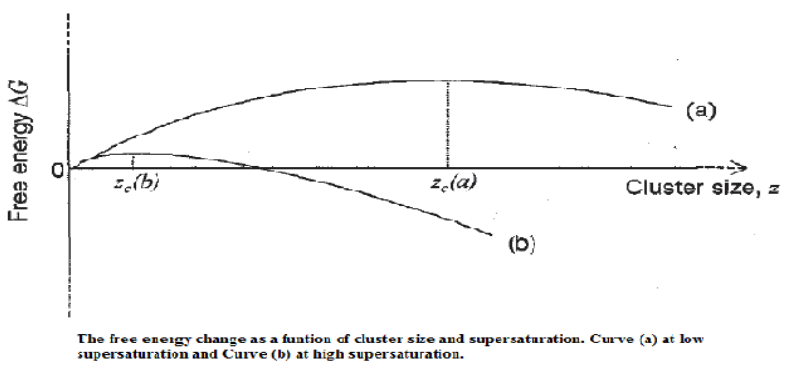

Fig. 4: free energy curve

Fig. 4 shows the variance of free energy with the cluster size. It gives an indication that the free energy at low supersaturation is less stable and at high supersaturation this free energy is stable for wide range of cluster size distribution. At $\mathrm{z}_{\mathrm{c}}$ (a) the value of free energy is maximum.

Another conclusion drawn is that the chemical potential in the bulk of the crystals is unaffected by the supersaturation i.e. the monomers don't feel the monomers in the solution. It is therefore equal to saturated chemical potential at equilibrium [6].

\subsection{Nucleation and Crystal growth}

Before moving on to the process of Nucleation, we first have to take into consideration an another process which is known as Ostwald Ripening. It is more favorable to add monomers to large crystals. The ground state is one infinite crystal. A system can lower its free energy by letting small crystals dissolve and adding the freed monomers to large crystals.

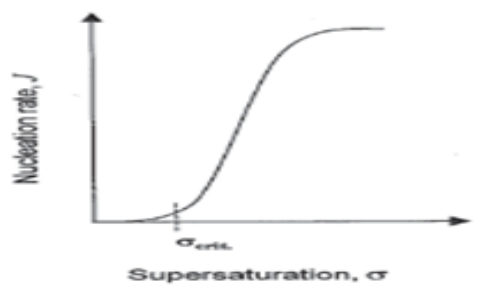

Fig. 5: nucleation vs supersaturation

Fig. 5 shows the rate of nucleation as a function of supersaturation. In the graph, it shows that the nucleation rate increases with the increase in the supersaturation after a certain critical supersaturation concentration which is $\sigma_{\text {crti. }}$ Up to this concentration there is no nucleation and as soon as the concentration of solute reaches this concentration the process of nucleation starts.

After the nuclei are formed the crystals will grow as long as the solution is supersaturated. If nucleation and growth are separated in time all growing crystals end up with very similar sizes since they have a similar history. The production of nuclei is stopped and only the preformed nuclei aggregate to form the larger and stable particles. This is popularly known as Lamer Model or "The separation of nucleation and growth".

\subsection{Growth rate}

When the growth is not so fast then the rate of growth is determined by the value of $\Delta \mathrm{G}$ which is referred to as Gibb's free energy. But when the growth is fast then it is determined not by $\Delta \mathrm{G}$ but by the rate of diffusion to the surface of the nanocrystal $[9,10]$. For fast growth, Fick's Law is taken into consideration which is explained below:-

Flux through surface of a sphere: - $\quad \mathrm{J}=4 \pi \mathrm{x}^{2} \mathrm{DdC} / \mathrm{dx}$

(where $\mathrm{D}=$ Diffusion Coefficient, $\mathrm{C}=$ concentration and $\mathrm{x}=$ position)

Total flux depends linearly on the radius as:- 
Nanoparticle Quantum Dots in Liquid phase (e.g. CdSe) - Synthesis, Thermodynamical study and

$\mathrm{J}=4 \pi \mathrm{D}\{(\mathrm{r}+\mathrm{d}) / \mathrm{d}\} \Delta \mathrm{C}$

(6)

(where $\mathrm{d}=$ length of diffusion layer, $\mathrm{r}=$ radius of sphere)

Relate flux and volume change:-

$\mathrm{J}=(\mathrm{dN} / \mathrm{dt})=\left(\mathrm{dV} / \mathrm{V}_{\mathrm{m}} \cdot \mathrm{dT}\right)=4 \pi D r \Delta C \quad(\mathrm{~d}>>\mathrm{r})$

(where $\mathrm{N}=$ number of molecules, $\mathrm{t}=$ Time and $\mathrm{V}_{\mathrm{m}}=$ volume)

Growth Rate inversely proportional to the radius:-

$\mathrm{dR} / \mathrm{dt}=\left(\mathrm{D} \cdot \mathrm{V}_{\mathrm{m}} \cdot \Delta \mathrm{C}\right) / \mathrm{r}$

Above presented equations via the Fick's law gives a range of conclusions. Increasing the radius of the crystal requires the incorporation of many more atoms than does increasing the radius of smaller crystals. The volume of the large crystals grows faster, but the radius grows slower. This is only true for high monomer concentrations. At low concentrations Ostwald Ripening dominates. As growth proceeds $\sigma$ drops and $r_{c}$ increases as per the formula obtained:-

$r_{c}=2 \Upsilon V_{m} / R T \sigma \quad$ (where $Y=$ constant)

Different important questions regarding the formation of CdSe nanoparticles can be answered by the above relation obtained i.e.

2.11.1 Why the temperature $\mathrm{T}$ taken to be high?

Precursors quickly decompose into reactive monomers, which lead to fast increase in supersaturation. Reaction is fast enough to stay in the focusing regime.

2.11.2 What is the role of the agent TOPO?

It is clear that they are used as capping agent to reduce the particles formed into the quantum dot range by influencing the surface energy, the chemical potential of the monomers and the rate of growth.

2.11.3 How is it possible that the crystals are monodisperse?

Crystals are monodisperse as a result of separation of nucleation and growth and the other reason is size focusing.

2.11.4 How long should the growth take?

The growth time should be short enough to stay in the size focusing regime ( $\sigma$ should be high) due to which high yields are impossible by definition.

\subsection{Applications}

These Quantum Dots of CdSe have wide applications in the field of Biomedical Research and other areas. Quantum dots are particularly significant for optical applications due to their theoretically high quantum yield. In Electronic applications they have been proven to operate like a single-electron transistor and show the Coulomb Blockade effect.

The ability to tune the size of quantum dots is advantageous for many applications. Larger quantum dots have a greater spectrum shift towards red compared to smaller dots, and exhibit less pronounced quantum properties. Conversely, the smaller particles give more subtle quantum effects. The new generation of Quantum dots has far reaching potential for the study of intracellular processes at the single molecule level, high resolution cellular imaging, long term in vivo observation of trafficking, tumor targeting and diagnostics.

Different fields have different applications as follows:-

Computational Field- It is preferably used in the solid state quantum computation. Quantum Dots can be used for the measurement of spin and other properties by controlling the flow of electrons through the quantum dots. Biological and Biotechnological Applications- Quantum Dots have two important properties i.e. of high quantum yield and they suffer less photo bleaching. Due to this they have high and long time brightness in comparison to the generally used dyes. Due to the highly improved photostablity of quantum dots many consecutive focal plane images can be reconstructed into high resolution 3-D images. This property also helps in real time tracking of the molecules. Semiconductor quantum dots have also been used for in vitro imaging of pre-labeled cells. Quantum Dots are dramatically better than existing methods for delivering a gene-silencing tool known as siRNA, into cells. Quantum Dots are also used as tumor targeting agents. Quantum dots are also used for the detection of cancer DNA by binding it in the presence of biotin protein.

Quantum Dot Lasers- These are the special kind of lasers which are more efficient then the general Quantum well or Semiconductor lasers. Due to their zero dimension and extremely small size there is approximately negligible loss and also less threshold current is required for the initiation of the process. Hence in the present day Quantum Dot Lasers are appeared to be efficient for the production of Laser. The disadvantage behind this is that the output power is very small due to small size, hence an array of quantum dots are used to obtain the desired output. 
Nanoparticle Quantum Dots in Liquid phase (e.g. CdSe) - Synthesis, Thermodynamical study and

Light Emitting Devices- "QD-LED displays" and "QD-WLED" (White LED) are being produced these days. Quantum dots are valued for displays because they emit light in very specific Gaussian distributions. This can result in a display that more accurately renders the colors that the human eye can perceive. Quantum dots also require very little power since they are not color filtered.

\section{Conclusions}

This paper concludes the overall study of nanoparticles in liquid phase and their thermodynamical study. It helps us to understand the formation of particles and the thermodynamic phenomena behind it. It also gives us a view regarding the energy concept behind the whole process of nucleation and growth. In short, it gives ample amount of knowledge about particles formation and their thermal stability.

\section{Acknowledgement}

I would like to express my gratitude to the Centre for Converging Technologies University of Rajasthan, Jaipur- India for providing me ample space and needs to perform this work. I am also thankful to Prof. Y.K.Vijay, University of Rajasthan, Jaipur-India, Prof. Ashok .K. Nagawat, University of Rajasthan, Jaipur-India for the support they have given me in the completion of my this effort.

Moreover I am thankful to my parents for their continuous support and encouragementLast but not the least I am thankful to the almighty God for its Blessing hand on me.

\section{References}

[1] X.G. Peng, L. Manna, W.D. Yang, J. Wickham, E. Scher, A. Kadavanich, A.P. Alivisatos, Nature 404 (2000) 59.

[2] Q. Peng, Y.J. Dong, Z.X. Deng, Y.D. Li, Inorg. Chem. 41 (2002) 5249.

[3] M.H. Chen, L. Gao, J. Am. Ceram. Soc. 88 (2005) 1643.

[4] L.F. Xi, Y.M. Lam, J. Colloid Interface Sci. 316 (2007) 771.

[5] M. Maillard, S. Giorgio, M.P. Pileni, Adv. Mater. 14 (2002) 1084.

[6] Y. Kawabata, M. Nagao, H. Seto, S. Komura, T. Takeda, D. Schwahn, N.L.Yamada, H. Nobutou, Phys. Rev. Lett. 92 (2004) 056103.

[7] X.Y. Ni, X.B. Su, Z.P. Yang, H.G. Zheng, J. Cryst. Growth 252 (2003) 612.

[8] I. Lisiecki, M.P. Pileni, J. Am. Chem. Soc. 115 (1993) 3887.

[9] G.D. Rees, R. Evans-Gowing, S.J. Hammond, B.H. Robinson, Langmuir 15 (1999) 1993.

[10] M.P. Pileni, Langmuir 17 (2001) 7476. 